\title{
NONLINEAR-PERIODIC CONTROL SYSTEM FOR NON-AFFINE MIMO PLANT WITH STATE DELAY
}

\author{
Eugenie L. Eremin \\ Informational and Control Systems Department \\ Amur State University \\ Russia \\ ereminel@mail.ru
}

Article history:

Received 01.11.2018, Accepted 19.12.2018

\begin{abstract}
The article deals with the synthesis problem of control system regulator for non-affine multivariable dynamic plant with state delay. The plant operates in periodic modes and also in the presence of external disturbances and parametric uncertainty. As a solution methods the hyperstability criterion, the fast-acting dynamic corrector, and $L$-dissipativity conditions are used. The key step of the system synthesis is the receipt for V. M. Popov's integral inequality special estimates that ensure the fulfillment of the control goals. In final article part with the help of simulation the dynamic processes taking place in the proposed control system, are being visually illustrated.
\end{abstract}

\section{Key words}

Control non-affine plant, priory uncertainty, nonlinear-periodic control, simulation, hyperstability criterion, $L$-dissipativity, filter-corrector, periodic signals generator.

\section{Introduction}

Nowadays the problems of developing control systems for various dynamic plants, which are operates in periodic modes, are still remaining relevant and indemand for modern control theory. This circumstance is due to the fact that similar automatic systems are very increasingly used on various practical solutions. For example, control of periodic modes may be occurring in: systems for manipulation robots [De Oliveira and Lages, 2016], some aircraft tracking systems [He, Guo, and Leang, 2017], systems for voltage converters [Li and Ye, 2018] and others.

It should be noted that often control plants are com-

\author{
Evgeniy A. Shelenok \\ Automation and Systems Engineering Department \\ Pacific National University \\ Russia \\ cidshell@mail.ru
}

plex multiply connected systems, the development of regulators for which requires special approaches. One such approach is the decentralized control method, in which the original plant is decomposed into several interconnected subsystems. And then for each local subsystem synthesis of control algorithms is carried out [Zhu and Pagilla, 2007], [Dragicevic, Wu, Shafiee, and Meng, 2017], [Shukla and Mili, 2017].

Many of modern publications are devoted to development of control laws for linear in control (affine) dynamic plants [Eilsen, Teo, and Fleming, 2017], [Shao and Xiang, 2017], [Yao, 2017]. At the same time, there are practically no works that are related to designing control systems for non-affine plants. However, plants mathematical models which contain nonlinear dependencies with respect to the input signal are encountered in a number of applied problems, such as: electromagnetic suspension control [Cho, Kato, and Spilman, 1993], underwater robots control [Pshikhopov and Medvedev, 2011] and aircraft control [Tavakol and Binazadeh, 2017]. In this regard, the analysis and synthesis problems of control algorithms for non-affine systems (including periodic ones) are very relevant and require careful consideration.

In this article, with the help of results obtained in [Eremin and Shelenok, 2017a], [Eremin and Shelenok, 2017b], [Eremin, 2013], [Eremin and Shelenok, 2015], the synthesis of periodic action decentralized control system for a stationary non-affine plant with state delay and maximum relative orders of local subsystems is being considered.

\section{Mathematical Description of the Control System}

We consider a control non-affine multi-loop dynamic plant which local subsystems mathematical model has 
the following form:

$$
\begin{aligned}
\frac{d x_{i}(t)}{d t}= & A_{i} x_{i}(t)+B_{i}\left[u_{i}(t) f_{i}\left(x_{i}(t), u_{i}(t)\right)+(1)\right. \\
& \left.+d_{i}^{T} x_{i}\left(t-\tau_{i}\right)+\varphi_{i}\left(u_{i}(t)\right)+\sigma_{i}(t)\right], \\
y_{i}(t)= & =x_{1_{i}}(t), \\
\sigma_{i}(t)= & \psi_{i}(t)+\sum_{j=1}^{k} \theta_{i j}(t), i=1,2, \ldots k ; i \neq j,
\end{aligned}
$$

where $x_{i}(t)=\left[x_{1_{i}}(t), x_{2_{i}}(t), \ldots, x_{n_{i}}(t)\right]^{T} \in R^{n_{i}}$ is the state vector of the $i$-th local subsystem; $A_{i}$ is some matrix in the Frobenius form; $B_{i}=\left[0, \ldots, 0, b_{n_{i}}\right] \in$ $\in R^{n_{i}}, b_{n_{i}}=$ const $>0$ is the stationary vector; $u_{i}(t) \in R$ and $y_{i}(t) \in R$ are respectively the scalar control signal and output; $d_{i}^{T}=\left[d_{1_{i}}, d_{2_{i}}, \ldots, d_{\left(n_{i}\right)_{i}}\right] \in$ $\in R^{n_{i}}$ is unknown constant vector; $\tau_{i}$ is the known constant delay; $f_{i}\left(x_{i}(t), u_{i}(t)\right)$ and $\varphi_{i}\left(u_{i}(t)\right)$ are smooth nonlinear scalar functions; $\sigma_{i}(t) \in R$ is the equivalent disturbance; $\psi_{i}(t) \in R$ is the periodic signal of constantly acting external disturbance; $\theta_{i j}(t) \in R$ is the cross couplings output signal acting on the $i$-th subsystem from the $j$-th subsystem side with dynamics

$$
\begin{aligned}
& \frac{d x_{i j}(t)}{d t}=A_{i j} x_{i j}(t)+B_{i j} y_{j}(t), \\
& \theta_{i j}(t)=L_{i j}^{T} x_{i j}(t), i \neq j,
\end{aligned}
$$

where $x_{i j}(t)=\left[x_{1_{i j}}(t), x_{2_{i j}}(t), \ldots, x_{\left(n_{i j}\right)_{i j}}(t)\right]^{T}$ is the cross coupling state vector; $A_{i j}, B_{i j}=[0, \ldots, 0,1]^{T}$, $L_{i j}^{T}$ are stationary matrix and vector respectively.

The control plant (??), (2) operates while the following assumptions are executed:

1. Parameters of the matrix $A_{i}$, vectors $B_{i}, d_{i}$ and the signals $f_{i}\left(x_{i}(t), u_{i}(t)\right), \varphi_{i}\left(u_{i}(t)\right)$ and $\psi_{i}(t)$ are priori unknowns and are determined by the relations:

$$
\begin{aligned}
& A_{i}=A_{i}(\xi), B_{i}=B_{i}(\xi), d_{i}=d_{i}(\xi) \\
& f_{i}\left(x_{i}(t), u_{i}(t)\right)=f_{\xi_{i}}\left(x_{i}(t), u_{i}(t)\right) \\
& \varphi_{i}(t)=\varphi_{\xi_{i}}(t), \psi_{i}(t)=\psi_{\xi_{i}}(t)
\end{aligned}
$$

where $\xi$ is unknown set of parameters belonging to a known bounded numerical set $\Xi$;

2. The subsystems (??) relative orders are exceed a single value.

3. The subsystems (??) relative orders are known and equal to $n_{i}$.

4. The internal states of the plant subsystems (??) are not available for measurement.

5. For direct measurement only signals $y_{i}(t)$ are available.
6. The nonlinear functions $f_{i}\left(x_{i}(t), u_{i}(t)\right)$, $\varphi_{i}\left(u_{i}(t)\right)$, and the signal $\psi_{i}(t)$ are satisfy the following expressions:

$$
\begin{aligned}
& \varepsilon_{1_{i}}<f_{i}\left(x_{i}(t), u_{i}(t)\right) \leq \varepsilon_{2_{i}} \\
& \left|\varphi_{i}(u(t))\right| \leq \varepsilon_{3_{i}},\left|\psi_{i}(t)\right|=\left|\psi_{i}(t+T)\right| \leq \varepsilon_{4_{i}}
\end{aligned}
$$

where $\varepsilon_{1_{i}}=$ const $>0, \varepsilon_{2_{i}}=$ const $>0, \varepsilon_{3_{i}}=$ $=$ const $>0$ and $\varepsilon_{4_{i}}=$ const $>0$ are known numbers;

7. Matrix $A_{i j}$ and vectors $B_{i j}, L_{i j}$ are determined in such a way that the transfer function of the cross couplings (2) corresponds to the stable dynamic unit like

$$
W_{i j}(s)=L_{i j}^{T}\left(s E_{i j}-A_{i j}\right)^{-1} B_{i j}=\frac{b_{i j}(s)}{c_{i j}(s)},
$$

where $s$ is a complex variable; $b_{i j}(s)$ and $c_{i j}(s)$ are Hurwitz polynomials; $E_{i j}$ is an identity matrix of the corresponding dimension.

8. The signals $\theta_{i j}(t)$ corresponding to the outputs of the dynamic cross couplings (2) and with regard for (4) the equivalents perturbations $\sigma_{\left(n_{i}\right)_{i}}(t)$ are bounded and satisfy the conditions

$$
\begin{aligned}
& \left|\sum_{j=1}^{k} \theta_{i j}(t)\right| \leq \tilde{\theta}_{0_{i j}}, \tilde{\theta}_{0_{i j}}=\text { const }>0, \\
& \left|\sigma_{i}(t)\right| \leq \tilde{\varepsilon}_{4_{i}}, \quad \tilde{\varepsilon}_{4_{i}}=\text { const }>0
\end{aligned}
$$

To define the desired dynamics of the plant at hand (??)-(6), we introduce in each its subsystem, similar [Eremin and Shelenok, 2017a] and [Eremin and Shelenok, 2017b], explicit local reference models

$$
\begin{aligned}
& \frac{d x_{M_{i}}(t)}{d t}=A_{M_{i}} x_{M_{i}}(t)+B_{M_{i}} r_{i}(t), \\
& y_{M_{i}}(t)=x_{M 1_{i}}(t), z_{M_{i}}(t)=g_{i}^{T} x_{M_{i}}(t),
\end{aligned}
$$

where $x_{M_{i}}(t)=\left[x_{M 1_{i}}(t), x_{M 2_{i}}(t), \ldots, x_{M\left(n_{i}\right)_{i}}(t)\right]^{T} \in$ $\in R^{n_{i}}$ is the reference state variables; $A_{M_{i}}$ is the Hurwitz matrix, last row of which has the form $\left[a_{M 1_{i}}, a_{M 2_{i}}, \ldots, a_{M\left(n_{i}\right)_{i}}\right], a_{M i_{i}}$ are known numbers; $B_{M_{i}}$ is the known vector, $r_{i}(t)=r_{i}(t+T)$ is the scalar periodic command signal; $y_{M_{i}}(t) \in R$ is the main local reference output (to define the required motion of the local subsystem); $z_{M_{i}}(t) \in R$ is the auxiliary local reference model output (to specify the dynamics for the local main control contour); $g_{i}^{T}=\left[1, g_{1_{i}}, g_{2_{i}}, \ldots, g_{\left(n_{i}-1\right)_{i}}\right]$ is the given vector. 
For control plant (??)-(6) and reference model (7), following structural matching conditions

$$
A_{M_{i}}=A_{i}+B_{M_{i}} C_{0_{i}}^{T}, B_{M_{i}} K_{0_{i}}=B_{i}
$$

where $C_{0_{i}}^{T}=\left[C_{01_{i}}, C_{02_{i}}, \ldots, C_{0\left(n_{i}\right)_{i}}\right] \in R^{n_{i}}, K_{0_{i}}$ are unknown constant vector and number respectively; are fulfilled.

Since only the outputs signals $y_{i}(t)$ are measured, we introduce in the each local main control loop a filtercorrector (see [Eremin and Shelenok, 2017b]) that has following mathematical description:

$$
\begin{aligned}
& \frac{d x_{F_{i}}(t)}{d t}=A_{F_{i}} x_{F_{i}}(t)+B_{F_{i}} y_{i}(t), \\
& z_{F_{i}}(t)=C_{F_{i}}^{T} x_{F_{i}}(t)+D_{F_{i}} y_{i}(t),
\end{aligned}
$$

where $x_{F_{i}}(t)=\left[x_{F 1_{i}}(t), \ldots, x_{F\left(n_{i}-1\right)_{i}}(t)\right]^{T} \in$ $\in R^{\left(n_{i}-1\right)}$ is the filter state variables vector; $z_{F_{i}}(t) \in$ $\in R$ is the filter output; $A_{F_{i}}, B_{F_{i}}, C_{F_{i}}, D_{F_{i}}$ are matrices and vector defined in such a way that transfer function of the filter can be represented as follows:

$$
\begin{aligned}
& W_{F_{i}}(s)=\frac{g_{i}(s)}{\left(T_{i} s+1\right)^{\left(n_{i}-1\right)}}= \\
& =C_{F_{i}}^{T}\left(s E_{\left(n_{i}-1\right)}-A_{F_{i}}\right)^{1} B_{F_{i}}+D_{F_{i}},
\end{aligned}
$$

where $g_{i}(s)$ is the polynomial which composed with respect to coefficients of vector $g_{i} ; T_{i}$ is a small time constant.

\section{Problem Statement}

For the non-affine plant (??)-(6) which operates under the uncertainty conditions (3), it is required to synthesize a control law

$$
u_{i}(t)=u_{i}\left(x_{i}(t), x_{i}\left(t-\tau_{i}\right), x_{F_{i}}(t), r_{i}(t)\right)
$$

which for any initial conditions $x_{i}(0)$ and any level of parametric uncertainty $\xi \in \Xi$ will provides the fulfillment of the following condition

$$
\begin{aligned}
& \lim _{t \rightarrow \infty}\left|y_{M_{i}}(t)-y_{i}(t)\right| \leq \Delta_{y_{i}}, \\
& \Delta_{y_{i}}=\text { const }>0
\end{aligned}
$$

where $\Delta_{y_{i}}$ is a small value.

\section{Synthesis of the Control Law}

Construction of the control system we will carry out in accordance with the two-stage methodology which is described in [Eremin and Shelenok, 2015], [Eremin and Shelenok, 2017a], and [Eremin and Shelenok, 2017b]. At the first stage, we will obtain an explicit form of the control law (11) in assumption of availability the internal states $x_{i}(t)$ of the subsystems (??), (2). To determine the explicit form of control law (11), it is used the standard scheme of V. M. Popov's hyperstability criterion. In the second stage, we will ensure the $L$-dissipativity of the synthesized system with the help of estimates $x_{F_{i}}(t)$ of the variables $x_{i}(t)$ and special conditions.

Using equations (8) and also the concept of mismatch the state variables of local reference models (7) and subsystems of the plant (??) $\left(e_{i}(t)=x_{M_{i}}(t)-x_{i}(t)\right)$, we represent the equivalent mathematical description of the studied system as follows

$\frac{d e_{i}(t)}{d t}=A_{M_{i}} e_{i}(t)+B_{M_{i}} \mu_{i}(t)$,

$v_{i}(t)=z_{M_{i}}(t)-g_{i}^{T} x_{i}(t)$,

$\mu_{i}(t)=-\left[u_{i}(t)-\delta_{i}(t)-C_{0}^{T} x_{i}(t)+\right.$

$+K_{0} d_{i}^{T} x_{i}\left(t-\tau_{i}\right)+\left(K_{0_{i}} f_{i}\left(x_{i}(t), u_{i}(t)\right)-1\right) u_{i}(t)+$

$\left.+K_{0_{i}} \varphi_{i}\left(u_{i}(t)\right)\right]$,

where $\delta_{i}(t)=r_{i}(t)-K_{0_{i}} \sigma_{i}(t)$ is a periodic signal.

For the linear stationary part $(L S P)$ of an equivalent system (13), it is necessary to ensure the validity of condition

$$
R e\left[W_{L S P_{i}}(j \omega)\right]>0, \forall \omega>0,
$$

where $W_{L S P_{i}}(j \omega)$ is the appropriate frequency transfer function; $j^{2}=-1$. It can be shown (see [Eremin and Shelenok, 2017a] and [Eremin and Shelenok, 2017b]) that, the choice of values of the vector $g_{i}$ elements, based on following relation

$$
\begin{aligned}
& s^{n_{i}}+a_{M\left(n_{i}\right)_{i}} s^{\left(n_{i}-1\right)}+\ldots+a_{M 2_{i}} s+a_{M 1_{i}}= \\
& =\left(s+a_{*_{i}}\right)\left(s^{\left(n_{i}-1\right)}+\frac{g_{\left(n_{i}-2\right)_{i}}}{g_{\left(n_{i}-1\right)_{i}}} s^{\left(n_{i}-2\right)}+\ldots+\right. \\
& \left.+\frac{g_{1_{i}}}{g_{\left(n_{i}-1\right)_{i}}} s+\frac{1}{g_{\left(n_{i}-1\right)_{i}}}\right) g_{\left(n_{i}-1\right)_{i}},
\end{aligned}
$$

where $a_{*_{i}}=$ const is an any root of the polynomial $a_{M_{i}}(s)$ which is written with respect to the coefficients $a_{M_{i}}, i=1,2, \ldots, n_{i}$; it is possible to ensure the existence of transfer function 


$$
\begin{aligned}
& W_{L S P_{i}}(j \omega)=g_{i}^{T}\left(j \omega E_{i}-A_{M_{i}}\right)^{-1} B_{M_{i}}= \\
& =K_{0_{i}} \frac{a_{*_{i}}}{a_{*_{i}}+j \omega},
\end{aligned}
$$

for which inequality (14) is always feasible.

Let us define the control signal $u_{i}(t)$ as a sum of five components:

$$
\begin{aligned}
& u_{i}(t)=\zeta_{1_{i}}(t)+\zeta_{2_{i}}(t)+\zeta_{3_{i}}(t)+ \\
& +\zeta_{4_{i}}(t)+\zeta_{5_{i}}(t) .
\end{aligned}
$$

In this case the expression corresponding to nonlinear non-stationary part of the equivalent system (13) is converted to the following form:

$$
\begin{aligned}
& \mu_{i}(t)=-\left[\left(\zeta_{1_{i}}(t)-\delta_{i}(t)\right)+\right. \\
& +\left(\zeta_{2_{i}}(t)-C_{0_{i}}^{T} x_{i}(t)\right)+ \\
& +\left(\zeta_{3_{i}}(t)+K_{0_{i}} d_{i}^{T} x_{i}\left(t-\tau_{i}\right)\right)+ \\
& +\left(\zeta_{4_{i}}(t)+\left(K_{0_{i}} f_{i}\left(x_{i}(t), u_{i}(t)\right)-1\right) u_{i}(t)\right)+ \\
& \left.+\left(\zeta_{5_{i}}+K_{0_{i}} \varphi\left(u_{i}(t)\right)\right)\right] .
\end{aligned}
$$

Let's satisfy the requirements of V. M. Popov's integral inequality

$$
\begin{aligned}
& \eta_{i}(0, t)=-\int_{0}^{t} \mu_{i}(\varsigma) v_{i}(\varsigma) d \varsigma \geq-\eta_{0_{i}}^{2} \\
& \eta_{0_{i}}=\text { const }, \forall t>0
\end{aligned}
$$

the left side of which, taking into account (16), we write as follows:

$$
\begin{aligned}
& \eta_{i}(0, t)=\sum_{j=1}^{5} \eta_{j_{i}}(0, t)= \\
& =\int_{0}^{t}\left[\zeta_{1_{i}}(\varsigma)-\delta_{i}(\varsigma)\right] v_{i}(\varsigma) d \varsigma+ \\
& +\int_{0}^{t}\left[\zeta_{2_{i}}(\varsigma)-C_{0_{i}}^{T} x_{i}(\varsigma)\right] v_{i}(\varsigma) d \varsigma+ \\
& +\int_{0}^{t}\left[\zeta_{3_{i}}(\varsigma)+K_{0_{i}} d_{i}^{T} x_{i}\left(\varsigma-\tau_{i}\right)\right] v_{i}(\varsigma) d \varsigma+ \\
& +\int_{0}^{t}\left[\zeta_{4_{i}}(\varsigma)+\left(K_{0_{i}} f_{i}\left(x_{i}(\varsigma), u_{i}(\varsigma)\right)-1\right) u_{i}(\varsigma)\right] \times \\
& \times v_{i}(\varsigma) d \varsigma+\int_{0}^{t}\left[\zeta_{5_{i}}(\varsigma)+K_{0_{i}} \varphi_{i}\left(u_{i}(\varsigma)\right)\right] v_{i}(\varsigma) d \varsigma
\end{aligned}
$$

In accordance with the results were obtained in [Eremin and Shelenok, 2015], we synthesize component $\zeta_{1_{i}}(t)$ of the control law (15) in the form

$$
\zeta_{1_{i}}(t)=\zeta_{1_{i}}\left(t-\bar{T}_{i}\right)+\gamma_{1_{i}} v_{i}(t),
$$

where $\gamma_{1_{i}}=$ const $>0, \bar{T}_{i}=$ const $>0$. Then for the integral summand $\eta_{1_{i}}(0, t)$ it will be fair the following estimate:

$$
\begin{aligned}
& \eta_{1_{i}}(0, t)= \\
& =\gamma_{1_{i}} \int_{0}^{t} v_{i}(\varsigma)\left[\int_{0}^{\varsigma} \omega_{0}(\varsigma-h) v_{i}(h) d h-\delta_{i}(\varsigma)\right] d \varsigma \geq \\
& \geq-\eta_{01_{i}}^{2}, \eta_{01_{i}}=\text { const }, \forall t>0,
\end{aligned}
$$

where $\omega_{0}(\cdot)$ is the weight function of periodic signals generator with transfer function $W(s)=$ $=\frac{\beta}{1-e^{-s \bar{T}}}, \beta=$ const $\geq 1$.

Considering the second integral term from (18):

$$
\begin{aligned}
& \eta_{2_{i}}(0, t)=\int_{0}^{t}\left[\zeta_{2_{i}}(\varsigma)-C_{0_{i}}^{T} x_{i}(\varsigma)\right] v_{i}(\varsigma) d \varsigma= \\
& =\int_{0}^{t}\left[\zeta_{2_{i}}(\varsigma)-\sum_{p=1}^{n_{i}} C_{0 p_{i}} x_{p_{i}}(t)\right] v_{i}(\varsigma) d \varsigma,
\end{aligned}
$$

we define the component $\zeta_{2_{i}}(t)$ like

$$
\begin{aligned}
& \zeta_{2_{i}}(t)=\sum_{p=1}^{n_{i}} \gamma_{2 p_{i}} x_{p_{i}}(t) \int_{0}^{t} x_{p_{i}}(\varsigma) v_{i}(\varsigma) d \varsigma, \\
& \gamma_{2 p_{i}}=\text { const }>0 .
\end{aligned}
$$

Then, taking into account the identity

$$
\int_{0}^{t} \chi(\varsigma) \int_{0}^{\varsigma} \chi(h) d h d \varsigma=0.5\left(\int_{0}^{t} \chi(\varsigma) d \varsigma\right)^{2}
$$

where signal $\chi(t)$ is bounded, it can be written following estimate:

$$
\begin{aligned}
& \eta_{2_{i}}(0, t)=\int_{0}^{t}\left[\sum_{p=1}^{n_{i}} \gamma_{2 p_{i}} x_{p_{i}}(\varsigma) \int_{0}^{\varsigma} x_{p_{i}}(\vartheta) v_{i}(\vartheta) d \vartheta-\right. \\
& \left.-\sum_{p=1}^{n_{i}} C_{0 p_{i}} x_{p_{i}}(\varsigma)\right] v_{i}(\varsigma) d \varsigma=\sum_{p=1}^{n_{i}} \gamma_{2 p_{i}} \int_{0}^{t} x_{p_{i}}(\varsigma) v_{i}(\varsigma) \times
\end{aligned}
$$


$\times \int_{0}^{\varsigma} x_{p_{i}}(\vartheta) v_{i}(\vartheta) d \vartheta d \varsigma-\sum_{p=1}^{n_{i}} C_{0 p_{i}} \int_{0}^{t} x_{p_{i}}(\varsigma) v_{i}(\varsigma) d \varsigma=$

$$
\begin{aligned}
& =0.5 \sum_{p=1}^{n_{i}} \gamma_{2 p_{i}}\left(\int_{0}^{t} x_{p_{i}}(\varsigma) v_{i}(\varsigma) d \varsigma\right)^{2}- \\
& \eta_{3_{i}}(0, t)=\sum_{p=1}^{n_{i}} \gamma_{3 p_{i}} \int_{0}^{t} x_{p_{i}}\left(\varsigma-\tau_{i}\right) v_{i}(\varsigma) \times \\
& \times \int_{0}^{\varsigma} x_{p_{i}}\left(\vartheta-\tau_{i}\right) v_{i}(\vartheta) d \vartheta d \varsigma- \\
& +\sum_{p=1}^{n_{i}} K_{0_{i}} d_{p_{i}} \int_{0}^{t} x_{p_{i}}\left(\varsigma-\tau_{i}\right) v_{i}(\varsigma) d \varsigma= \\
& -\sum_{p=1}^{n_{i}} C_{0 p_{i}} \int_{0}^{t} x_{p_{i}}(\varsigma) v_{i}(\varsigma) d \varsigma \pm \sum_{p=1}^{n_{i}} \frac{C_{0 p_{i}}^{2}}{2 \gamma_{2 p_{i}}}= \\
& =0.5 \sum_{p=1}^{n_{i}} \gamma_{3 p_{i}}\left(\int_{0}^{t} x_{p_{i}}\left(\varsigma-\tau_{i}\right) v_{i}(\varsigma) d \varsigma\right)^{2}+ \\
& +\sum_{p=1}^{n_{i}} K_{0_{i}} d_{p_{i}} \int_{0}^{t} x_{p_{i}}\left(\varsigma-\tau_{i}\right) v_{i}(\varsigma) d \varsigma \pm \\
& \pm \sum_{p=1}^{n_{i}} \frac{\left(K_{0_{i}} d_{p_{i}}\right)^{2}}{2 \gamma_{3 p_{i}}}=\left[0.5 \sum_{p=1}^{n_{i}} \gamma_{3 p_{i}} \times\right. \\
& \times\left(\int_{0}^{t} x_{p_{i}}\left(\varsigma-\tau_{i}\right) v_{i}(\varsigma) d \varsigma\right)^{2}+ \\
& +\sum_{p=1}^{n_{i}} K_{0_{i}} d_{p_{i}} \int_{0}^{t} x_{p_{i}}\left(\varsigma-\tau_{i}\right) v_{i}(\varsigma) d \varsigma+ \\
& \left.-\sum_{p=1}^{n_{i}} C_{0 p_{i}} \int_{0}^{t} x_{p_{i}}(\varsigma) v_{i}(\varsigma) d \varsigma+\sum_{p=1}^{n_{i}} \frac{C_{0 p_{i}}^{2}}{2 \gamma_{2 p_{i}}}\right]- \\
& \left.\sum_{p=1}^{n_{i}} \frac{\left(K_{0_{i}} d_{p_{i}}\right)^{2}}{2 \gamma_{3 p_{i}}}\right]-\sum_{p=1}^{n_{i}} \frac{\left(K_{0_{i}} d_{p_{i}}\right)^{2}}{2 \gamma_{3 p_{i}}} \geq \\
& \geq-\sum_{p=1}^{n_{i}} \frac{\left(K_{0_{i}} d_{p_{i}}\right)^{2}}{2 \gamma_{3 p_{i}}}=-\eta_{03_{i}}^{2}, \eta_{03_{i}}=\text { const, } \forall t>0 . \\
& -\sum_{p=1}^{n_{i}} \frac{C_{0 p_{i}}^{2}}{2 \gamma_{2 p_{i}}} \geq-\sum_{p=1}^{n_{i}} \frac{C_{0 p_{i}}^{2}}{2 \gamma_{2 p_{i}}}=-\eta_{02_{i}}^{2}
\end{aligned}
$$

Let's transform the term $\eta_{4_{i}}(0, t)$ as follows:

$$
\eta_{02_{i}}=\text { const, } \forall t>0
$$

Let us define the component $\zeta_{3_{i}}(t)$ as follows

$$
\begin{array}{ll} 
& \eta_{4_{i}}(0, t)=\int_{0}^{t}\left[\zeta_{4_{i}}(\varsigma)+\left(K_{0_{i}} f_{i}\left(x_{i}(\varsigma), u_{i}(\varsigma)\right)-1\right) \times\right. \\
\zeta_{3_{i}}(t)=\sum_{p=1}^{n_{i}} \gamma_{3 p_{i}} x_{p_{i}}\left(t-\tau_{i}\right) \times \quad(21) & \left.\times u_{i}(\varsigma)\right] v_{i}(\varsigma) d \varsigma=\int_{0}^{t} \zeta_{4_{i}}(\varsigma) v_{i}(\varsigma) d \varsigma+ \\
\times \int_{0}^{t} x_{p_{i}}\left(\varsigma-\tau_{i}\right) v(\varsigma) d \varsigma, \gamma_{3 p_{i}}=\text { const }>0 . & \\
& +\int_{0}^{t}\left(K_{0_{i}} f_{i}\left(x_{i}(\varsigma), u_{i}(\varsigma)\right)-1\right) u_{i}(\varsigma) v_{i}(\varsigma) d \varsigma \pm \\
& \pm 2 \bar{\gamma}_{4_{i}}^{2} \tilde{\gamma}_{4_{i}} \int_{0}^{t} u_{i}(\varsigma) v_{i}(\varsigma) \int_{0}^{\varsigma} u_{i}(\vartheta) v_{i}(\vartheta) d \vartheta d \varsigma \pm \\
\begin{array}{ll}
\text { Then for summand } \eta_{3_{i}}(0, t) \text { we obtain following esti- } \\
\text { mate: }
\end{array} & \pm \frac{1}{4 \tilde{\gamma}_{4_{i}}} \geq \int_{0}^{t}\left[\zeta_{4_{i}}(\varsigma)-2 \bar{\gamma}_{4_{i}}^{2} \tilde{\gamma}_{4_{i}} u_{i}(\varsigma) \times\right.
\end{array}
$$
mate: 


$$
\begin{aligned}
& \left.\times \int_{0}^{\varsigma} u_{i}(\vartheta) v_{i}(\vartheta) d \vartheta\right] v_{i}(\varsigma) d \varsigma+\left[\tilde{\gamma}_{4_{i}} \times\right. \\
& \times\left(\int_{0}^{t}\left(K_{0_{i}} f_{i}\left(x_{i}(\varsigma), u_{i}(\varsigma)\right)-1\right) u_{i}(\varsigma) v_{i}(\varsigma) d \varsigma\right)^{2}+ \\
& +\int_{0}^{t}\left(K_{0_{i}} f_{i}\left(x_{i}(\varsigma), u_{i}(\varsigma)\right)-1\right) u_{i}(\varsigma) v_{i}(\varsigma) d \varsigma+ \\
& \left.+\frac{1}{4 \tilde{\gamma}_{4_{i}}}\right]-\frac{1}{4 \tilde{\gamma}_{4_{i}}},
\end{aligned}
$$

where $\bar{\gamma}_{4_{i}}=\max \left|K_{0_{i}} f_{i}\left(x_{i}(t), u_{i}(t)\right)-1\right|=$ $=$ const $>0 ; \tilde{\gamma}_{4_{i}}=$ const $>0$. If now we synthesized the component $\zeta_{4_{i}}(t)$ in the form

$$
\begin{aligned}
& \zeta_{4_{i}}(t)=\gamma_{4_{i}} u_{i}(t) \int_{0}^{t} u_{i}(\varsigma) v_{i}(\varsigma) d \varsigma \\
& \gamma_{4_{i}}=2 \bar{\gamma}_{4_{i}}^{2} \tilde{\gamma}_{4_{i}}=\text { const }>0
\end{aligned}
$$

for summand $\eta_{4_{i}}(0, t)$ we will have a fair estimate

$$
\eta_{4_{i}}(0, t) \geq-\frac{1}{4 \tilde{\gamma}_{4_{i}}}=-\eta_{04_{i}}^{2}, \eta_{04_{i}}=\text { const, } \forall t>0
$$

Let us define the explicit form of the component $\zeta_{5_{i}}(t)$ in the following form:

$$
\zeta_{5_{i}}(t)=\gamma_{5_{i}} \int_{0}^{t} v_{i}(\varsigma) d \varsigma
$$

where $\gamma_{5_{i}}=2 \tilde{\gamma}_{5_{i}} \varepsilon_{3_{i}}^{2}=$ const $>0$.

In this case for the summand $\eta_{5_{i}}(0, t)$ it will be fair the following estimate:

$$
\begin{aligned}
& \eta_{5_{i}}(0, t)=\int_{0}^{t}\left[\zeta_{5_{i}}(\varsigma)+K_{0_{i}} \varphi_{i}\left(u_{i}(\varsigma)\right)\right] v_{i}(\varsigma) d \varsigma \pm \\
& \pm 2 \tilde{\gamma}_{5_{i}} \varepsilon_{3_{i}}^{2} \int_{0}^{t} v_{i}(\varsigma) \int_{0}^{\varsigma} v_{i}(\vartheta) d \vartheta d \varsigma \geq \\
& \geq \int_{0}^{t}\left[\zeta_{5_{i}}(\varsigma)-2 \tilde{\gamma}_{5_{i}} \varepsilon_{3_{i}}^{2} \int_{0}^{\varsigma} v_{i}(\vartheta) d \vartheta\right] v_{i}(\varsigma) d \varsigma+ \\
& +\tilde{\gamma}_{5_{i}}\left(\int_{0}^{t} \varphi_{i}\left(u_{i}(\varsigma)\right) v_{i}(\varsigma) d \varsigma\right)^{2}+ \\
& +K_{0_{i}} \int_{0}^{t} \varphi_{i}\left(u_{i}(\varsigma)\right) v_{i}(\varsigma) d \varsigma \pm \frac{K_{0_{i}}^{2}}{4 \tilde{\gamma}_{5_{i}}} \geq-\frac{K_{0_{i}}^{2}}{4 \tilde{\gamma}_{5_{i}}}=\eta_{05_{i}}^{2} \\
& \eta_{05_{i}}=\text { const, } \forall t>0 .
\end{aligned}
$$

Thus, mathematical description of the regulator (11), which does not contradict the validity of (17), in accordance with (19), (20), (21), (22) and (23), will take the form

$$
\begin{aligned}
& u_{i}(t)=\left(\zeta_{1_{i}}\left(t-\bar{T}_{i}\right)+\gamma_{1_{i}} v_{i}(t)\right)+ \\
& +\sum_{p=1}^{n_{i}} \gamma_{2 p_{i}} x_{p_{i}}(t) \int_{0}^{t} x_{p_{i}}(\varsigma) v_{i}(\varsigma) d \varsigma+ \\
& +\sum_{p=1}^{n_{i}} \gamma_{3 p_{i}} x_{p_{i}}\left(t-\tau_{i}\right) \int_{0}^{t} x_{p_{i}}\left(\varsigma-\tau_{i}\right) v_{i}(\varsigma) d \varsigma+ \\
& +\gamma_{4_{i}} u_{i}(t) \int_{0}^{t} u_{i}(\varsigma) v_{i}(\varsigma) d \varsigma+\gamma_{5_{i}} \int_{0}^{t} v_{i}(\varsigma) d \varsigma
\end{aligned}
$$

where $v_{i}(t)=z_{M_{i}}(t)-g_{i}^{T} x_{i}(t)$. Since the frequency condition (14) is satisfied, and also the valid integral inequality (17) exists, the equivalent system (13), (15), (19)-(24) and, consequently, the control system (??), (2), (4)-(7), (9)-(11), (24) will be hyperstable, and for this system an auxiliary $\lim _{t \leftarrow \infty}\left|v_{i}(t)\right| \leq \Delta_{z_{i}}, \Delta_{z_{i}}=$ $=$ const $>0$ and, the main (12) functioning targets are going to be fair.

\section{L-dissipativity of the Control System}

The control law synthesis was carried out on the assumption of the full availability of the local state vectors $x_{i}(t)$. But for direct measurements, only the outputs signals of the plant local subsystems $y_{i}(t)$ are available. So, for technical realization of the obtained nonlinear-periodic algorithm (24), it is necessary to use the estimations of the variables $x_{i}(t)$, which are the state variables of the filter-corrector (9), (10). It should be noted that the filter parameters must be specified in a certain way [Eremin, 2013]. In particular, values of the time constants $T_{i}$ (see (10)) we should choose with the help of special conditions:

$$
\begin{aligned}
T_{i}<T_{1_{i}} & =\frac{0.93}{\left(n_{i}-2\right) a_{M 1_{i}}} \\
T_{i}<T_{2_{i}} & =\frac{0.465 \cdot a_{M 1_{i}}}{\left(n_{i}-1\right) a_{M 2_{i}}}
\end{aligned}
$$

Thus, replacing in (24) the plant's subsystems state variables $x_{p_{i}}(t)$ by their estimates, we obtain following technically realizable control law:

$$
\begin{gathered}
u_{i}(t)=\left(\zeta_{1_{i}}\left(t-\bar{T}_{i}\right)+\gamma_{1_{i}} \bar{v}_{i}(t)\right)+ \\
+\sum_{p=0}^{n_{i}-1} \gamma_{2 p_{i}} \tilde{x}_{(p+1)_{i}}(t) \int_{0}^{t} \tilde{x}_{(p+1)_{i}}(\varsigma) \bar{v}_{i}(\varsigma) d \varsigma+ \\
+\sum_{p=0}^{n_{i}-1} \gamma_{3 p_{i}} \tilde{x}_{(p+1)_{i}}\left(t-\tau_{i}\right) \times
\end{gathered}
$$




$$
\begin{aligned}
& \times \int_{0}^{t} \tilde{x}_{(p+1)_{i}}\left(\varsigma-\tau_{i}\right) \bar{v}_{i}(\varsigma) d \varsigma+ \\
& +\gamma_{4_{i}} u_{i}(t) \int_{0}^{t} u_{i}(\varsigma) \bar{v}_{i}(\varsigma) d \varsigma+\gamma_{5_{i}} \int_{0}^{t} \bar{v}_{i}(\varsigma) d \varsigma \\
& \bar{v}_{i}(t)=z_{M_{i}}(t)-z_{F_{i}}(t)=z_{M_{i}}(t)-g_{i}^{T} x_{F_{i}}(t), \\
& -3.2 \leq a_{1_{1}} \leq 2.1,-1.5 \leq a_{2_{1}} \leq 1, \\
& -12 \leq a_{3_{1}} \leq 5.2,1 \leq b_{3_{1}} \leq 5.5,1.2 \leq d_{0} \leq 3.2 \text {, } \\
& 0 \leq d_{1_{1}} \leq 20,0.5 \leq d_{2_{1}} \leq 5.5,0.5 \leq d_{3_{1}} \leq 10.2 \\
& 0.02 \leq \varphi_{0_{1}} \leq 1,0 \leq \psi_{0_{1}} \leq 0.8 \\
& -10 \leq a_{1_{2}} \leq 5,-5 \leq a_{2_{2}} \leq 5.7,-1 \leq a_{3_{2}} \leq 15 \text {, } \\
& 0.1 \leq b_{3_{2}} \leq 3,2.2 \leq d_{1_{2}} \leq 18,0.1 \leq d_{2_{2}} \leq 7.3 \text {, } \\
& 0 \leq d_{3_{2}} \leq 25.4,0.02 \leq \varphi_{0_{2}} \leq 2.5,0 \leq \psi_{0_{2}} \leq 1.2 \text {, }
\end{aligned}
$$

where $\tilde{x}_{i}(t)=\left[x_{F 1_{i}}(t), x_{F 2_{i}}(t), \ldots, \dot{x}_{F\left(n_{i}-1\right)_{i}}(t)\right] \in$ $\in R^{n_{i}}$

In this case, the control system (??), (2), (4)-(7), (9)(11), (25) is going to be $L$-dissipative and will preserve operability in a given class of uncertainty $\xi \in \Xi$.

\section{Illustrative Example}

To illustrate the quality of synthesized system, let us consider the control problem of multi-loop plant, which consist of two subsystems with the following structure:

$$
\begin{aligned}
& A_{1}=\left(\begin{array}{ccc}
0 & 1 & 0 \\
0 & 0 & 1 \\
a_{1_{1}} & a_{2_{1}} & a_{3_{1}}
\end{array}\right), B_{1}=\left(\begin{array}{c}
0 \\
0 \\
b_{3_{1}}
\end{array}\right), \\
& d_{1}^{T}=\left(d_{1_{1}} d_{2_{1}} d_{3_{1}}\right), \\
& f_{1}\left(x_{1}(t), u_{1}(t)\right)=\frac{d_{0}}{1+\left|u_{1}(t)\right|^{2}}+1.5\left(1+x_{2_{1}}^{2}(t)\right), \\
& \varphi_{1}\left(u_{1}(t)\right)=\varphi_{0_{1}} \sin \left(0.5 u_{1}(t)\right), \psi_{1}(t)=\psi_{0_{1}} \sin (0.3 t) ;
\end{aligned}
$$

$$
A_{2}=\left(\begin{array}{ccc}
0 & 1 & 0 \\
0 & 0 & 1 \\
a_{1_{2}} & a_{2_{2}} & a_{3_{2}}
\end{array}\right), B_{2}=\left(\begin{array}{c}
0 \\
0 \\
b_{3_{2}}
\end{array}\right),
$$

$d_{2}^{T}=\left(\begin{array}{lll}d_{1_{2}} & d_{2_{2}} & d_{3_{2}}\end{array}\right)$,

$f_{2}\left(x_{2}(t), u_{2}(t)\right)=\frac{1}{0.25+u_{2}^{2}(t)}+1.1\left(1+0.1 x_{3_{2}}^{3}(t)\right)$, $\varphi_{2}\left(u_{2}(t)\right)=\varphi_{0_{2}} \sin \left(0.4 u_{2}(t)\right), \psi_{2}(t)=\psi_{0_{2}} \sin (0.4 t) ;$

$$
\begin{aligned}
& W_{12}(s)=\frac{s^{2}+2 s+1}{2 s^{3}+4 s^{2}+3 s+1} \\
& W_{21}(s)=\frac{s+1}{s^{2}+2 s+1}
\end{aligned}
$$

The class of a priori uncertainty of the plant (??), (26), (27) is determined by following inequalities:

The structure of the matrix and vectors of the local reference models we define in the following form:

$$
\begin{aligned}
A_{M_{i}} & =\left(\begin{array}{ccc}
0 & 1 & 0 \\
0 & 0 & 1 \\
a_{M 1_{i}} & a_{M 2_{i}} & a_{M 3_{i}}
\end{array}\right), \\
B_{M_{i}} & =\left(\begin{array}{c}
0 \\
0 \\
b_{M_{i}}
\end{array}\right), g_{i}=\left(\begin{array}{c}
1 \\
g_{1_{i}} \\
g_{2_{i}}
\end{array}\right), \\
a_{M 1_{i}} & =-20, a_{M 2_{i}}=-41, a_{M 3_{i}}=-22, \\
b_{M 3_{i}} & =23, g_{1_{i}}=2, g_{2_{i}}=1, i=1,2 ;
\end{aligned}
$$

wherein the local filter-correctors (9) are formed as follows:

$$
\begin{aligned}
& A_{F_{i}}=\left(\begin{array}{cc}
0 & 1 \\
a_{F 1_{i}} & a_{F 2_{i}}
\end{array}\right), B_{F_{i}}=\left(\begin{array}{c}
0 \\
b_{F 2_{i}}
\end{array}\right), \\
& C_{F_{i}}=\left(\begin{array}{c}
1 \\
g_{1_{i}}
\end{array}\right), D_{F_{i}}=g_{2_{i}}, \\
& a_{F 1_{i}}=-10^{6}, a_{F 2_{i}}=-2 \cdot 10^{3}, \\
& b_{F 2_{i}}=10^{6}, g_{1_{i}}=2, g_{2_{i}}=1, i=1,2 .
\end{aligned}
$$

The command signals of the subsystem were specified using following periodic functions:

$$
\begin{aligned}
& r_{1}(t)=0.9 \sin ^{5}(0.1 t) \cdot(2-\sin (0.05 t)) \\
& r_{2}(t)=0.5 \sin ^{2}(0.1 t) \cdot(\sin (0.05 t)-1)
\end{aligned}
$$

Simulation of the control system (??)-(9), (26)-(31) was performed at the following plant coefficients:

$$
\begin{aligned}
& a_{1_{1}}=0.2, a_{2_{1}}=-1, a_{3_{1}}=-6, \\
& d_{1_{1}}=2, d_{2_{1}}=0.5, d_{3_{1}}=1, \\
& d_{0}=2, \varphi_{0_{1}}=0.2, \psi_{0_{1}}=0.5, \tau_{1}=2 ; \\
& d_{1_{2}}=3, d_{2_{2}}=0.1, d_{3_{2}}=1, \\
& a_{1_{2}}=-7.1, a_{2_{2}}=2, a_{3_{2}}=-0.5, \\
& \varphi_{0_{2}}=0.4, \psi_{0_{2}}=0.2, \tau_{2}=1 .
\end{aligned}
$$




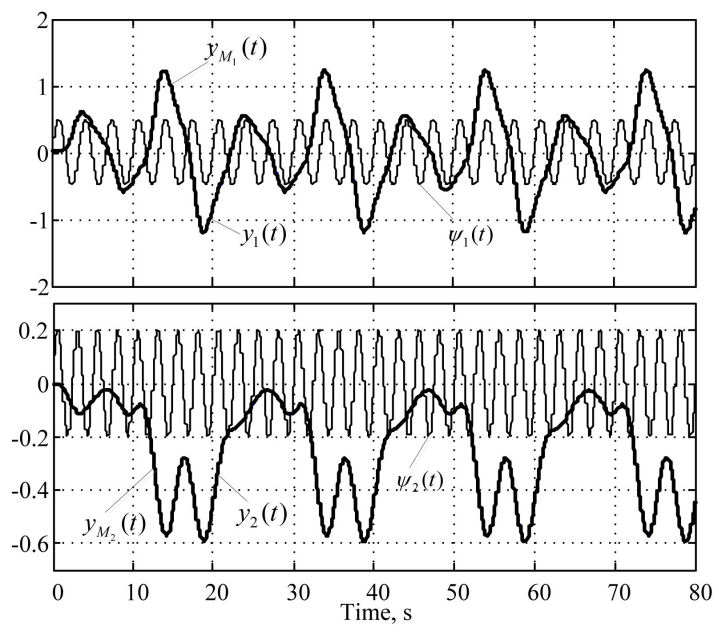

Figure 2. Dynamics of main outputs of the reference models $y_{M_{i}}(t)$, outputs of the subsystems $y_{i}(t)$ and the external perturbations $\psi_{i}(t)(i=1,2)$
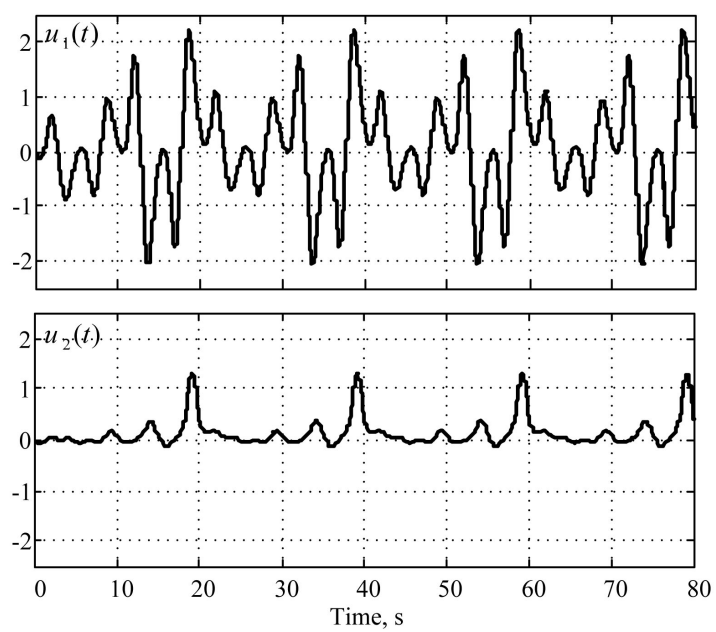

Figure 3. Local control signals

In the course of simulation parameters of the nonlinear-periodic regulator (25) in order to increase the system performance were selected as follows:

$$
\begin{aligned}
& \gamma_{1_{1}}=\gamma_{1_{2}}=100, \gamma_{21_{1}}=\gamma_{21_{2}}=500 \\
& \gamma_{22_{1}}=\gamma_{22_{2}}=600, \gamma_{23_{1}}=\gamma_{23_{2}}=400 \\
& \gamma_{31_{1}}=\gamma_{31_{2}}=300, \gamma_{32_{1}}=\gamma_{32_{2}}=200 \\
& \gamma_{33_{1}}=\gamma_{33_{2}}=400, \gamma_{4_{1}}=\gamma_{4_{2}}=200 \\
& \gamma_{5_{1}}=\gamma_{5_{2}}=200, \bar{T}_{1}=12, \bar{T}_{2}=10
\end{aligned}
$$

The results of the system simulation are shown at Fig. 1-3.

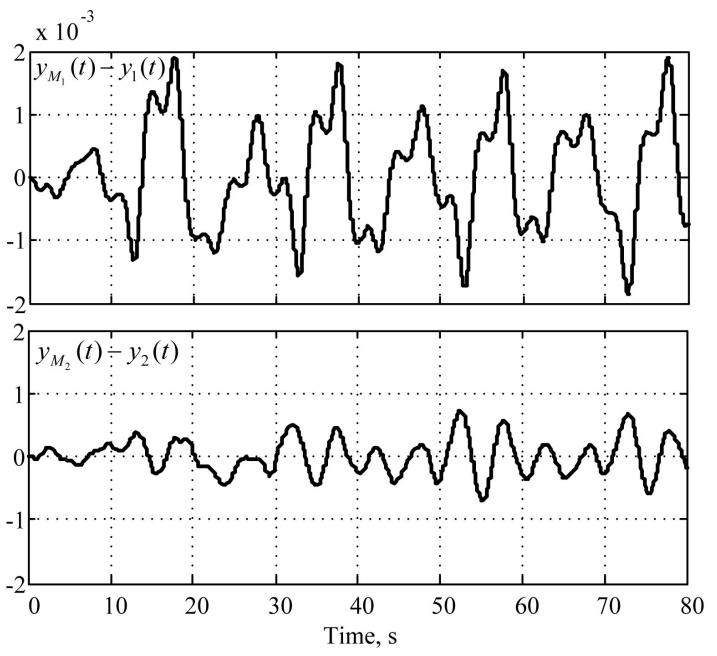

Figure 1. Mismatch signal between main outputs of the local reference models (29), and outputs of control plant subsystems (??), (26), (27)

The presented results make it possible to conclude that proposed control system (??)-(9), (26)-(33) has a quite high quality: the magnitude of control error from the start of operation does not exceed $0.16 \%$ to the both subsystems(Fig. 1). It means that the signals $y_{M_{i}}(t)$ and $y_{i}(t)$ are almost coincide (Fig. 2).

\section{Conclusion}

The synthesis method of the control system for one class of non-affine MIMO dynamic plants with delay is proposed. With the help of simulation it is shown that the resulting control law allows to achieve high quality of control system operation. The obtained results can be useful for construction the decentralized control systems for non-affine periodic action plants.

\section{Acknowledgements}

The work was supported by the Russian Foundation for Basic Research (project 17-08-00871).

\section{References}

Cho, D., Kato, Y. and Spilman, D. (1993) Sliding mode and classical controllers in magnetic levitation systems. IEEE Control Systems Magazine, 13: 42-47.

Oliveira, I.G. De and Lages, W.F. (2016). Repetitive Control Applied to Robot Manipulators. In Proc. 2016 IEEE 21 st Int. Conf. on Emerging Technologies and Factory Automation (ETFA), Germany, Berlin, Sept. 6-9.

Dragicevic, T., Wu, D., Shafiee, Q., and Meng, L. (2017). Distributed and Decentralized Control Architectures for Converter-Interfaced Microgrids. Chinese Journal of Electrical Engineering, 3(2): 41-52. 
Eilsen, A.A., Teo, Y.R., and Fleming, A.J. (2017). Improving Robustness Filter Bandwidth in Repetitive Control by Considering Model Mismatch. Asian Journal of Control, 19(4): 1-11.

Eremin, E.L. (2013). L-dissipativnost of hyperstable control system in structural indignation. IV. Information Science and Control Systems, 2(36): 100-106.

Eremin, E.L. and Shelenok, E.A. (2015). Adaptive Periodic Servo-System for Nonlinear Control-Affine Objects. Optoelectronics, Instrumentation and Data Processing, 51(5):523-529.

Eremin, E.L. and Shelenok, E.A. (2017). Nonlinear Robust Control System for an Unsteady Nonaffine Dynamic Plant with a Delay. Optoelectronics, Instrumentation and Data Processing, 53(2): 151-158.

Eremin, E.L. and Shelenok, E.A. (2017). Robust Control for One Class of Multivariable Dynamic Plants. Automation and Remote Control, 78(6): 1046-1058.

He, X., Guo, D., and Leang, K.K. (2017). Repetitive Control Design and Implementation for Periodic Motion Tracking in Aerial Robots. Proc. 2017 American Cont. Conf, USA, Seattle, May 24-26.

D. Li and Y. Ye. Phase Compensation Second-Order
Repetitive Control for CVCF PWM Inverters. Int. J. of Electronics, Vol. 105, Issue 2:324-336, 2018.

Pshikhopov, V.Kh. and Medvedev, M.Yu. (2011). Synthesis of control systems for underwater vehicles with nonlinear actuators. Izvestiya SFedU. Engineering Sciences, 3(116): 147-156.

Shao, J. and Xiang, Z. (2017). High-Order Repetitive Control for Discrete-Time Linear Switched Systems. Int. J. of System Science, 48(9): 1882-1890.

Shukla, S. and Mili, L. (2017). Hierarchical Decentralized Control for Enhanced Rotor Angle and Voltage Stability of Large-Scale Power Systems. IEEE Transactions on Power Systems, 32(6): 1-11.

Tavakol, F. and Binazadeh, T. (2017). Robust Control Design for Path Tracking of Non-Affine UAV. Systems Science \& Control Engineering, 5(1): 474-480. Yao, W.-S. (2017). Repetitive Control Design for Variable Instantaneous Frequencies Signals. Int. J. of Control, Automation and Systems, 15(2): 689-697.

Zhu, Y. and Pagilla, P.R. (2007). Decentralized output feedback control of a class of large-scale interconnected systems. IMA Journal of Mathematical Control and Information, 24: 57-69. 